\title{
Competition between Fusion and Quasifission in the Formation of Super-heavy Elements
}

\author{
David J. Hinde* \\ Department of Nuclear Physics, Research School of Physics and Engineering, \\ Australian National University, Canberra, ACT 2601, Australia \\ E-mail: david.hindedanu.edu.au
}

M. Dasgupta, D.Y. Jeung, G. Mohanto E. Prasad C. Simenel, E.C. Simpson, A. Wakhle E. Williams, I.P. Carter, K.J. Cooke, Sunil Kalkal II, D.C. Rafferty, J. Walshe Department of Nuclear Physics, Research School of Physics and Engineering, Australian National University, Canberra, ACT 2601, Australia

\begin{abstract}
Quasifission is a non-equilibrium dynamical process resulting in rapid separation of the dinuclear system initially formed after capture of two colliding heavy nuclei. This can inhibit fusion by many orders of magnitude, thus suppressing the cross section for formation of superheavy elements. Measurements with projectiles from $\mathrm{C}$ to Ni, made at the Australian National University Heavy Ion Accelerator Facility, have mapped out quasifission characteristics and systematics using mass-angle distributions (MAD) - the fission mass-split as a function of centre-of-mass angle. These provide information on quasifission dynamics in the least model-dependent way. Quasifission time-scale information in the MAD has been compared with TDHF calculations of the collisions, with good agreement being found. Most significantly, the nuclear structure of the two colliding nuclei has a dramatic effect on quasifission probabilities and characteristics in gentle collisions at near-barrier energies. The effect of static deformation alignment, closed shells and N/Z matching can completely change reaction outcomes. The realization of this strong dependence makes modelling quasifission and superheavy element formation a challenging task, but should ultimately allow more reliable prediction of superheavy element formation cross sections.
\end{abstract}

The 26th International Nuclear Physics Conference

11-16 September, 2016

Adelaide, Australia

\footnotetext{
${ }^{*}$ Speaker.

† Current address: BARC, Mumbai, India

${ }^{\ddagger}$ Permanent address: Department of Physics, School of Mathematical and Physical Sciences, Central University of Kerala, Kasaragod 671314, India.

${ }^{\S}$ Current address: National Superconducting Cyclotron Laboratory, Michigan State University, East Lansing, Michigan 48824, USA

${ }^{\mathbb{I}}$ Current address: School of Physics and Material Sciences, Thapar University, Patiala-147001, Punjab, India
} 
Superheavy elements (SHE) are formed by heavy-ion fusion reactions. Their cross sections can be considerably suppressed [1] by quasifission [2]. This non-equilibrium process results when the combined di-nuclear system, formed as the two nuclear surfaces stick together, subsequently separates into two (fission-like) fragments, with the initial kinetic energy largely or completely damped. Quasifission can occur very rapidly, typically in less than $10^{-20} \mathrm{~s}$, well-before a compact compound nucleus can be reached $[2,3,4]$. The probability of quasifission $\left(\mathrm{P}_{Q F}\right)$ can be very large, thus the complementary probability of compound nucleus formation $\left(\mathrm{P}_{C N}=1-\mathrm{P}_{Q F}\right)$ can be small, probably lower than $10^{-3}$ in reactions forming superheavy elements. Understanding the competition between quasifission and fusion is thus very important in predicting the optimal fusion reactions to use to form new elements and isotopes in the superheavy mass region.

A key quantity characterizing the non-equilibrium quasifission process is the "sticking time" between contact of the two nuclear surfaces [5] and breakup (scission). Since the two colliding nuclei always approach each other along the beam axis, and after contact rotate with angular velocities that can be calculated, measurement of the rotation angle allows estimation of the sticking time. As sketched in Fig.1, as the system rotates, mass flow also occurs between the two nuclei. Measurements of mass over a wide range of angles is called a mass-angle distribution (MAD). This gives the most direct information on the dynamics, as long as the system undergoes less than a full rotation (taking $\sim 10^{-20} \mathrm{~s}$ ). This is usually the case for collisions of heavy nuclei, as shown by first measurements at GSI [2, 6], and later results from ANU [4, 7, 8, 9, 10, 3, 11, 12, 13]. The kinematic coincidence technique used in the measurements $[2,4,14]$ provides direct information on the mass-ratio of the fragments at scission; thus, the data are represented in terms of mass ratio $\mathrm{M}_{R}$, rather than pre- or post-evaporation masses, which cannot be determined by this method without

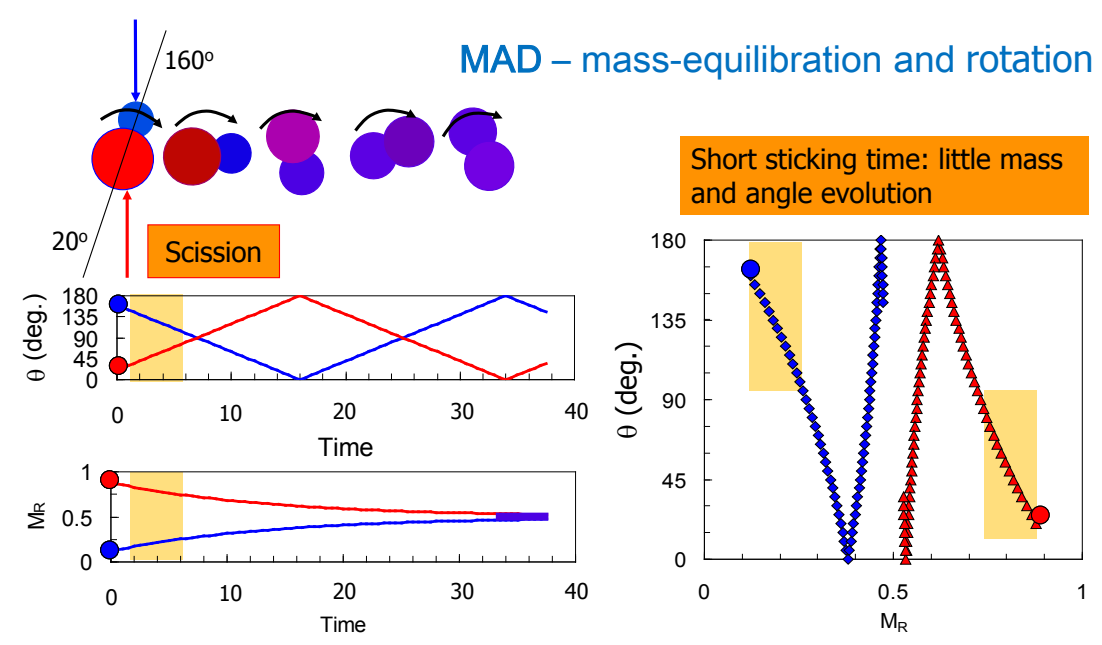

Figure 1: The simple principles underlying the mass-angle distribution (MAD) are illustrated. The sketches at top left show the sequence of configuration changes after initial contact of light (blue) and heavy (red) nuclei, here with an initial centre-to-centre angle of $160^{\circ}$. The system rotates and equilibrates in mass (or mass-ratio $\mathrm{M}_{R}$ ) as a function of time (arbitrary units), as shown below. Zeptosecond times cannot be measured directly, however mass-ratio and angle can be measured. One trajectory in mass-ratio and angle is illustrated at bottom right, the orange highlighted region indicating the collision outcomes resulting from a short sticking time. 

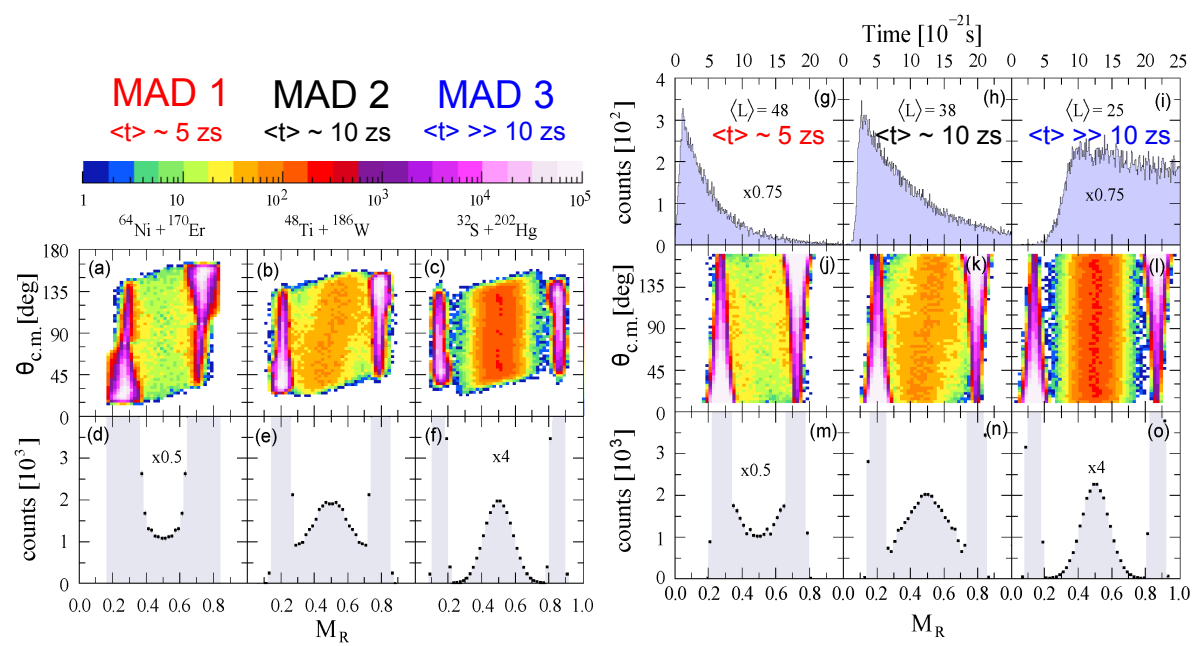

Figure 2: Panels (a)-(c) show the experimental MADs, with (d)-(f) showing the projections onto $M_{R}$, for the reactions ${ }^{64} \mathrm{Ni}+{ }^{170} \mathrm{Er},{ }^{48} \mathrm{Ti}+{ }^{186} \mathrm{~W}$ and ${ }^{32} \mathrm{~S}+{ }^{202} \mathrm{Hg}$, which all form ${ }^{234} \mathrm{Cm}$. The multiplicative factors shown scale the y-axis. The simulated MADs for the same reactions and beam energies are shown in panels (j),(k),(l), with the corresponding $M_{R}$ spectra in panels (m),(n),(o), and the sticking time distributions used for the simulations in $(\mathrm{g}),(\mathrm{h}),(\mathrm{i})$, where the calculated mean angular momentum following capture, and the deduced approximate mean sticking times are also given (adapted from Ref. [12]).

making assumptions.

Measured MAD and deduced quasifission sticking time distributions are shown in Fig. 2, for reactions forming the compound nucleus ${ }^{234} \mathrm{Cm}$ [12]. The MAD can be most simply categorised [4] by their distinctly different characteristics: (i) minimum mass yield at symmetry associated with short sticking times; (ii) a mass-angle correlation with peak yield at symmetry; and (iii) no significant mass-angle correlation associated with long sticking times. These are assigned the categories MAD1, MAD2 and MAD3 respectively [4]. By choosing bombarding energies above the capture barrier, the systematic behaviour of MAD with the indentity of the two colliding nuclei was studied systematically [4], to determine the global trends of quasifission dynamics. This is in analogy with the evaluation of the smooth liquid drop model dependence of nuclear masses on $\mathrm{N}$ and $\mathrm{Z}$, which highlights the effects of nuclear structure on nuclear masses. It was shown [4] that there is a strong correlation between the MAD type and the entrance channel fissility, and also the compound nucleus fissility. However, for particular cases, as discussed below, it has been found that nuclear structure of the nuclei in the entrance channel is extremely important in determining the sticking times and MAD characteristics. Shell structure including static deformation [10] and spherical magic numbers [11] can be very important, as well as overall neutron richness [11, 15]. These should be understood quantitatively to assess implications for future reactions aiming to synthesise new superheavy elements and isotopes.

The effect of static deformation alignment of the deformed heavy reaction partner is shown clearly in the dependence of the MAD (and corresponding mass-width [16]) as the beam energy transitions from above to below the average capture barrier energy. This is illustrated in Fig. 3 for reactions of ${ }^{48} \mathrm{Ti}$ with a range of heavy nuclei [16]. These extend from ${ }^{144} \mathrm{Sm}$ (spherical) through ${ }^{154} \mathrm{Sm},{ }^{174} \mathrm{Yb},{ }^{192} \mathrm{Os}$ (all prolate deformed) to ${ }^{208} \mathrm{~Pb}$ (spherical). For deformed nuclei, at energies 


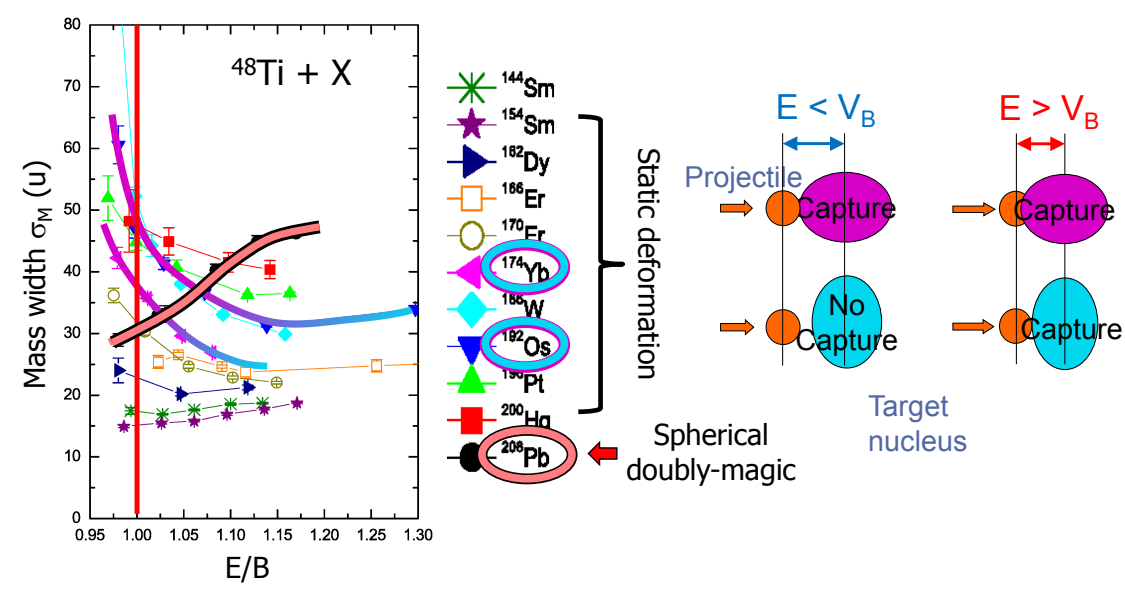

Figure 3: The left panel shows the mass-width (converted from mass-ratio) for the different target nuclei indicated. It is plotted as a function of the ratio of the c.m. kinetic energy $E$ of the ${ }^{48} \mathrm{Ti}$ projectile to the capture barrier energy $B$. For the reactions of heavy, statically deformed nuclei, the mass width rises rapidly as the energy falls across the barrier. In contrast, the heavy spherical doubly-magic nucleus ${ }^{208} \mathrm{~Pb}$ shows the opposite behaviour (see text). The sketches on the right illustrate that at energies below $B$, only deformationaligned collisions result in contact and capture, whilst at high $E / B$, the distance of closest approach is smaller (red arrow), and capture also occurs for compact configurations which give longer sticking times.

below the average barrier $B$, the reduced Coulomb repulsion at contact [5] only permits elongated (deformation aligned) configurations to result in capture, illustrated schematically on the right side of Fig.3. The implications for the subsequent quasifission are discussed below. The broad mass widths seen in Fig. 2 at below-barrier energies $(\mathrm{E} / \mathrm{B}<1)$ correspond to MAD (not shown) of type 2 (see Fig.2), and thus correspond to sticking times of $\sim 10 \mathrm{zs.} \mathrm{At} \mathrm{above-barrier} \mathrm{energies,}$ the reducing mass width is associated with increasing contributions from the compact equatorial contact configurations, having longer sticking times, and mass distributions closer to symmetry. Because of the higher Coulomb energy at contact, equatorial configurations can only contribute to the observed quasifission at energies above the average capture barrier.

In reactions with much heavier prolate deformed actinide nuclei such as ${ }^{238} \mathrm{U}$, the difference between the elongated, deformation-aligned contact configurations seen exclusively below-barrier, and the compact equatorial collisions is even greater. This is illustrated in Fig.4, for the reaction ${ }^{40} \mathrm{Ca}+{ }^{238} \mathrm{U}$. The sub-barrier energy shows a MAD of type 1, with time scale $\sim 5 \mathrm{zs}$. Above-barrier, mass-symmetric events become increasingly probable, here too associated with compact contact configurations and longer sticking times. Microscopic quantal mean-field TDHF calculations [13] were carried out for this reaction with two extreme orientations: deformation-aligned (axial) and equatorial collisions. The equatorial collisions (for all non-zero impact parameter) had a sticking time typically $6 \mathrm{zs}$, almost independent of angular momentum. Similarly, the Z, N and A of the quasifission fragments from axial collisions showed little variation with angular momentum, excitation energy, or with sticking time, being centred on 82, 122 and 204 respectively. This suggests that the Z,N closed shells around ${ }^{208} \mathrm{~Pb}$ are important in the TDHF calculations. As shown in Fig.4, the TDHF calculations of mass-ratios and fragment angles for axial collisions show good agreement with the experimental MAD, implying shell effects are indeed playing an important role in 


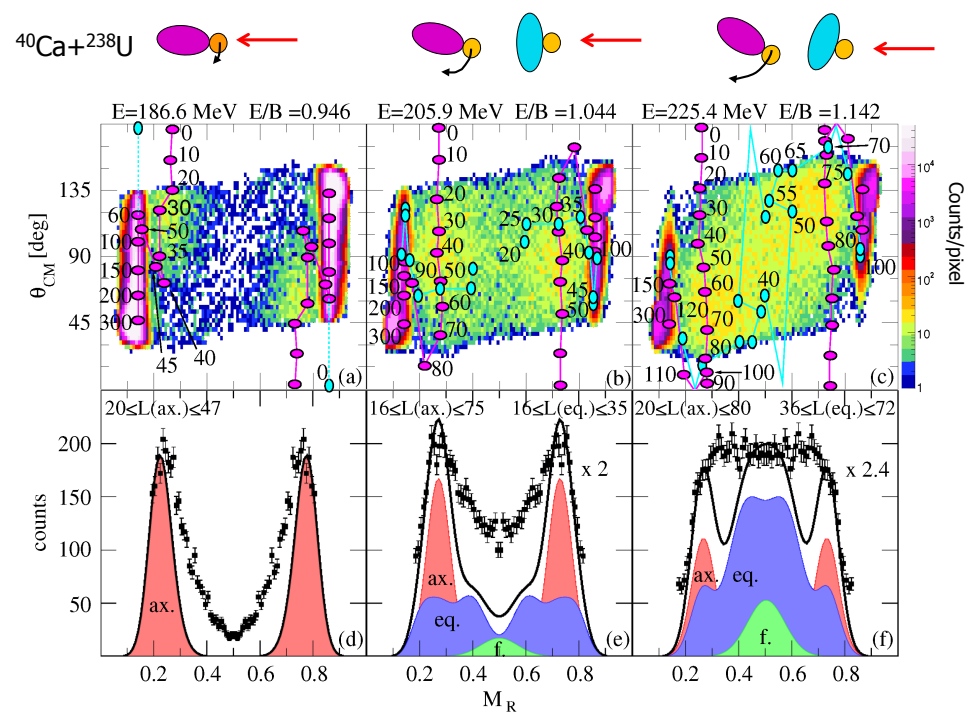

Figure 4: Comparison of experimental MAD (see pixel count colour scale) and mass-ratio spectra (below) with the mass-ratio and angle outcome of TDHF calculations for axial (purple ellipses) and equatorial (blue) collisions at the indicated angular momenta (from Ref. [13]). Comparisons from below-barrier ( $\mathrm{E} / \mathrm{B}=0.946$ ) to above-barrier $(\mathrm{E} / \mathrm{B}=1.142)$ show TDHF agrees well with experiment, axial collisions having short sticking times and little mass-evolution, in contrast with equatorial collisions, which at low angular momenta remain as one nucleus, assumed to eventually undergo mass-symmetric fission (green: f.).

quasifission. In the calculations, equatorial collisions for low impact parameters do not re-separate within 25-40 zs, and are assumed to lead to fusion, and subsequent fusion-fission. The higher impact parameters have sticking times and mass-ratios strongly dependent on angular momentum. For a given sticking time, the equatorial collisions generally experience more mass-equilibration than the axial collisions, again suggesting the special role of the ${ }^{208} \mathrm{~Pb}$ double closed shell in the axial collisions. However, the experimental mass-ratio distributions for the mass-asymmetric splits are quite broad, extending smoothly towards mass-symmetry, as seen in the bottom left panel in Fig. 4. This is not consistent with the potential energy surface as a function of mass-asymmetry, which shows a well-defined valley around $\mathrm{Pb}$. Thus it may be that the effect of the ${ }^{208} \mathrm{~Pb}$ structure on the mass distributions may have a dynamical origin, and the mechanism, including the effect of fluctuations, deserves deeper investigation.

The heaviest element $(\mathrm{Z}=118)$ that can be formed in fusion reactions using ${ }^{48} \mathrm{Ca}$ has already been created [17], and recently named Oganesson. To create heavier elements by fusion, heavier projectiles must be used. A key question in superheavy element synthesis is whether SHE cross sections will be drastically smaller in fusion reactions using projectiles heavier than ${ }^{48} \mathrm{Ca}$. To address this question indirectly, measurements of quasifission MADs for a range of projectile and target combinations (including ${ }^{48} \mathrm{Ca}$ ) are underway at the ANU. These will show how the average behaviour in these collisions changes with the identity of the projectile and target nuclei. First results are available for the ${ }^{54} \mathrm{Cr}+{ }^{238} \mathrm{U}$ reaction. MAD measurements from sub-barrier $(\mathrm{E} / \mathrm{B}=0.93)$ to well above barrier $(\mathrm{E} / \mathrm{B}=1.16)$ are shown in Fig. 5. At sub-barrier energies, the MAD look very similar to those for ${ }^{40} \mathrm{Ca}+{ }^{238} \mathrm{U}$ shown in Fig. 4, with short sticking times and little mass evolution. Above-barrier, for instance at $\mathrm{E} / \mathrm{B}=1.04$, increasing the projectile charge by 4 compared with 


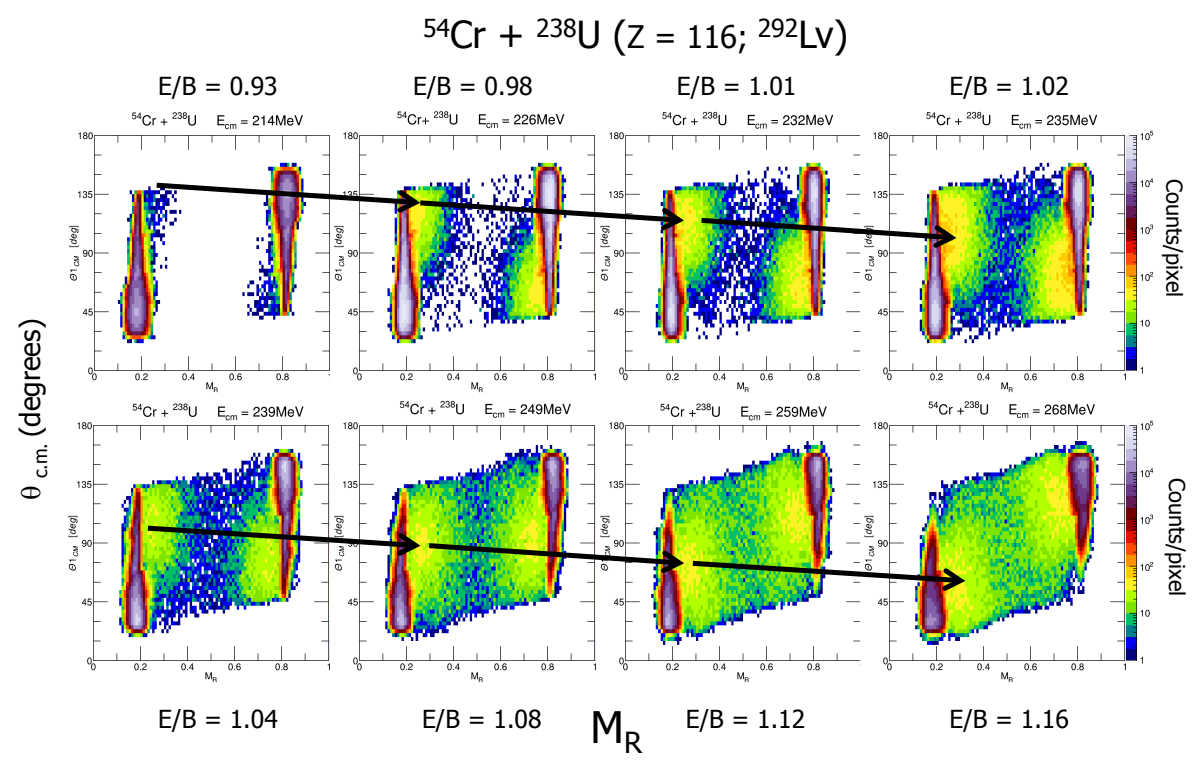

Figure 5: $\mathrm{MAD}$ for the ${ }^{54} \mathrm{Cr}+{ }^{238} \mathrm{U}$ reaction, from $\mathrm{E} / \mathrm{B}=0.93$ to $\mathrm{E} / \mathrm{B}=1.16$. The arrows indicate the smooth evolution in c.m. angle of the mass-asymmetric component as the beam energy increases.

the MAD shown in Fig. 4 significantly reduces the fraction of events resulting in mass-symmetric splits. Instead most of the events still result in fast mass-asymmetric outcomes which were previously identified (for lighter projectiles) as resulting from deformation-aligned, or axial collisions. The mass-ratio of this group of events changes little with beam energy, and the angle of peak yield moves forwards consistent with increasing angular momentum input. On evaluation of the angular momentum distributions from the cross sections, the quasifission time scales will be extracted from the MAD for each projectile. This will show whether the average behaviour for ${ }^{48} \mathrm{Ca}$ is very different from the heavier projectiles, (which could then be associated with its doubly magic and neutron-rich properties), or whether the observations show a smooth evolution with projectile charge. The latter would imply that heavier projectile nuclei might provide adequate SHE cross sections, with the lightest possible projectile likely to provide the largest cross sections.

Sub-barrier reactions with (spherical) closed-shell nuclei show contrasting behaviour to reactions with heavy statically deformed nuclei. This is highlighted in Fig. 3, where the mass width for the ${ }^{48} \mathrm{Ti}+{ }^{208} \mathrm{~Pb}$ reaction falls as the beam energy drops, in contrast to neighbouring deformed, non-magic nuclei, where it rises steeply. To investigate in detail the effect on quasifission of closed shells in the entrance channel, measurements [11] of MAD were made for ${ }^{40,44,48}$ Ca projectiles bombarding targets of ${ }^{208,204} \mathrm{~Pb}$ (forming ${ }^{248,252} \mathrm{No}$ with $\mathrm{Z}_{\text {C.N. }}=102$ ), and for ${ }^{48} \mathrm{Ti}$ bombarding ${ }^{200} \mathrm{Hg}$ $\left({ }^{248} \mathrm{No}\right)$ and ${ }^{208} \mathrm{~Pb}\left({ }^{256} \mathrm{Db}\right.$ with $\left.\mathrm{Z}_{C . N .}=104\right)$. Measurements were made a few percent below the average fusion barrier energy to ensure low relative velocity at contact. The MAD and projected $\mathrm{M}_{R}$ spectra are shown in Fig. 6, together with reference data for the ${ }^{16} \mathrm{O}+{ }^{238} \mathrm{U}$ reaction at an above-barrier energy, forming ${ }^{254} \mathrm{Fm}$. The Gaussian fit standard deviations $\sigma_{M R}$, with experimental uncertainties, and the $\sigma_{M R}$ value for ${ }^{48} \mathrm{Ca}+{ }^{208} \mathrm{~Pb}$ from Ref. [18] are tabulated. Despite having similar or identical $\mathrm{Z}_{1} \mathrm{Z}_{2}$, and forming similar or identical compound nuclei, there is a wide variation in the $\sigma_{M R}$ values, indicating a significant variation in the probability/characteristics of quasifis- 


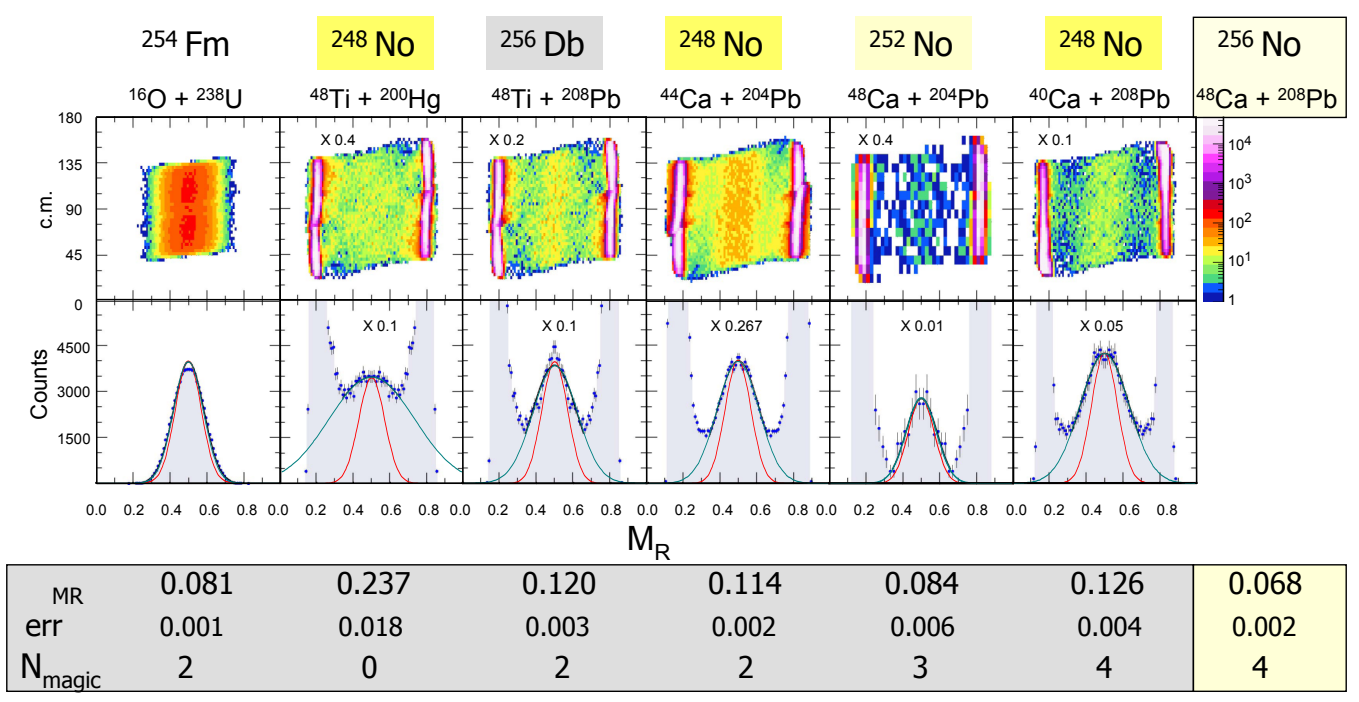

Figure 6: Measured mass-angle distributions for each reaction (upper panels). The factor multiplies the maximum counts of the logarithmic color scale (right). In the projected mass ratio spectra (lower panels) the scale factor multiplies the counts scale on the left. Gaussian fits to the region around $M_{R}=0.5$ are shown (turquoise lines), whose standard deviations $\sigma_{M R}$ are given below. Gaussian functions with $\sigma_{M R}=0.07$ (thin red lines) are shown for reference.

sion. Indeed, for the ${ }^{48} \mathrm{Ca}+{ }^{208} \mathrm{~Pb}$ reaction, measured mass distributions [18] appear consistent with a fusion-fission mechanism. To understand this, the correlation of $\sigma_{M R}$ with the number of magic numbers $\mathrm{N}_{m}$ in the entrance channel is informative [11]. The strong correlation results in $\sigma_{M R}$ approaching the expectation for fusion-fission $\left(\sigma_{M R}=0.07\right)$ with maximal $\mathrm{N}_{m}$ for ${ }^{48} \mathrm{Ca}+{ }^{208} \mathrm{~Pb}$. This suggests that reactions involving nuclei having several magic numbers form a compact true compound nucleus with higher probability. It seems likely that this is associated with reduced energy dissipation as the two nuclei overlap, allowing more compact shapes to be reached. These results led to the conclusion that "magicity" plays its strongest role when the N/Z values of the projectile and target nuclei are well-matched. When this is the case, transfer reactions that destroy entrancechannel magicity (as in the ${ }^{40} \mathrm{Ca}+{ }^{208} \mathrm{~Pb}$ reaction [11]) are minimized, preserving the closed shell nature of the collision partners as long as possible during the merging of the two nuclei [11]. Supporting this hypothesis, systematic analysis of xn evaporation residue cross sections in reactions forming Th isotopes has suggested enhanced fusion probabilities for two magic numbers in the entrance channel [19].

More recent ANU quasifission results, for the reaction of isotopes of $\mathrm{Cr}$ with $\mathrm{Pb}$, give the same conclusions regarding magicity and N/Z matching. MAD and mass-ratio projections at a subbarrier energy for each reaction studied are shown in Fig. 7. The left three reactions all form the compound nucleus ${ }^{258} \mathrm{Sg}$. These measurements, at sub-barrier energies, result in the low excitation energies (above the ground-state) as indicated, and shell effects may still have a strong influence on the reaction dynamics. They support the conclusions drawn above from the $\mathrm{Ca}+\mathrm{Pb}$ data. The difference between the N/Z values of the target and projectile nuclei is denoted by $\Delta_{N / Z}$ in the figure. The panels are ordered from left to right first by the number of magic numbers in the entrance channel $\left(\mathrm{N}_{M}\right)$, and then by $\Delta_{N / Z}$. The left-most reaction has only a single magic number in 


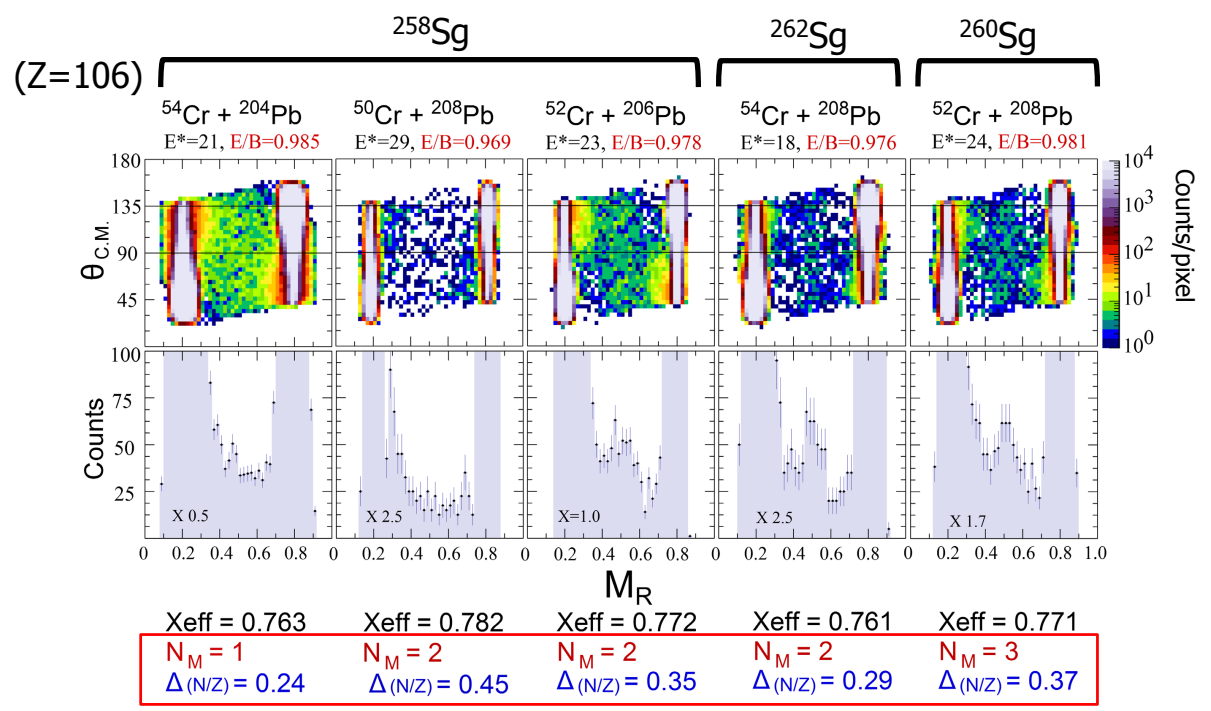

Figure 7: Experimental MAD and projected mass-ratio spectra $\left(90^{\circ}<\theta_{c . m .}<135^{\circ}\right)$ for sub-barrier $(\mathrm{E} / \mathrm{B} \sim 0.98)$ collisions of ${ }^{50,52,54} \mathrm{Cr}$ isotopes with ${ }^{204,206,208} \mathrm{~Pb}$, resulting in low excitation energies $\mathrm{E}^{*}$ as indicated. The entrance channel fissility $x_{e f f}$ [4], the total number of magic numbers of the projectile and target nuclei $\left(\mathrm{N}_{M}\right)$ and the difference between their N/Z ratios $\left(\Delta_{N / Z}\right)$ is indicated for each reaction. More magic numbers gives an angle-independent mass-symmetric fission component, suggestive of fusion-fission, as long as the $\Delta_{N / Z}$ value is not too large. N/Z mismatch is expected to result in transfer reactions that will break the initial magic numbers [11].

the entrance channel, and shows a U-shaped mass distribution, consistent with MAD class 1. With two magic numbers, the reactions better matched in N/Z $\left(\Delta_{N / Z}\right.$ closer to zero) show a peak at masssymmetry, associated with an angle-independent ridge in the MAD. With three magic numbers, but less favourable $\Delta_{N / Z}$, a similar result is observed. As the beam energy is increased, this masssymmetric peak becomes a smaller and smaller fraction of the total fission yield within the range $0.3<\mathrm{M}_{R}<0.7$. These reactions show a very similar behaviour to the ${ }^{40,44,48} \mathrm{Ca}+{ }^{204,208} \mathrm{~Pb}$ reactions; however, the transition from a U-shaped mass distribution to a narrow mass-symmetric component in the mass distribution is an even more drastic change in reaction outcome, and strongly suggests a significant bifurcation in reaction trajectories that is less obvious in the $\mathrm{Ca}+\mathrm{Pb}$ MADs. The very sudden change in outcome, depending on neutron number and magicity, will be a severe challenge for models of quasifission to reproduce. And yet it is this level of sensitivity that models must strive to reproduce, to optimise experimental opportunities to create new isotopes of superheavy elements. Investigating the relationship between these changing quasifission characteristics and changes in the heavy element yields themselves would be of great value.

\section{References}

[1] D. J. Hinde, M. Dasgupta and A. Mukherjee, Severe inhibition of fusion by quasifission in reactions forming ${ }^{220} \mathrm{Th}$, Phys. Rev. Lett. 89 (Dec, 2002) 282701.

[2] J. Tōke, B. Bock, G. X. Dai, A. Gobbi, S. Gralla, K. D. Hildenbrand et al., Quasi-fission - the mass-drift mode in heavy-ion reactions, Nucl. Phys. A 440 (1985) 327-365. 
[3] R. du Rietz, D. J. Hinde, M. Dasgupta, R. G. Thomas, L. R. Gasques, M. Evers et al., Predominant time scales in fission processes in reactions of $s$, ti and ni with $w$ : Zeptosecond versus attosecond, Phys. Rev. Lett. 106 (Feb, 2011) 052701.

[4] R. du Rietz, E. Williams, D. J. Hinde, M. Dasgupta, M. Evers, C. J. Lin et al., Mapping quasifission characteristics and timescales in heavy element formation reactions, Phys. Rev. C 88 (Nov, 2013) 054618.

[5] M. Dasgupta, D. J. Hinde, N. Rowley and A. M. Stefanini, Measuring barriers to fusion, Annu. Rev. Nucl. Part. Sci. 48 (1998) 401-461.

[6] W. Q. Shen, J. Albinski, A. Gobbi, S. Gralla, K. D. Hildenbrand, N. Herrmann et al., Fission and quasifission in u-induced reactions, Phys. Rev. C 36 (1987) 115 - 142.

[7] R. G. Thomas, D. J. Hinde, D. Duniec, F. Zenke, M. Dasgupta, M. L. Brown et al., Entrance channel dependence of quasifission in reactions forming ${ }^{220} \mathrm{Th}$, Phys. Rev. C 77 (Mar, 2008) 034610.

[8] R. Rafiei, R. G. Thomas, D. J. Hinde, M. Dasgupta, C. R. Morton, L. R. Gasques et al., Strong evidence for quasifission in asymmetric reactions forming ${ }^{202} \mathrm{Po}$, Phys. Rev. C 77 (Feb, 2008) 024606.

[9] D. J. Hinde, R. du Rietz, M. Dasgupta, R. G. Thomas and L. R. Gasques, Two distinct quasifission modes in the ${ }^{32} \mathrm{~S}+{ }^{232} \mathrm{Th}$ reaction, Phys. Rev. Lett. 101 (Aug, 2008) 092701.

[10] D. J. Hinde, R. G. Thomas, R. du Rietz, A. Diaz-Torres, M. Dasgupta, M. L. Brown et al., Disentangling effects of nuclear structure in heavy element formation, Phys. Rev. Lett. 100 (May, 2008) 202701.

[11] C. Simenel, D. J. Hinde, R. du Rietz, M. Dasgupta, M. Evers, C. J. Lin et al., Influence of entrance-channel magicity and isospin on quasi-fission, Phys. Lett. B 710 (2012) 607.

[12] E. Williams, D. J. Hinde, M. Dasgupta, R. du Rietz, I. P. Carter, M. Evers et al., Evolution of signatures of quasifission in reactions forming curium, Phys. Rev. C 88 (Sep, 2013) 034611.

[13] A. Wakhle, C. Simenel, D. J. Hinde, M. Dasgupta, M. Evers, D. H. Luong et al., Interplay between quantum shells and orientation in quasifission, Phys. Rev. Lett. 113 (Oct, 2014) 182502.

[14] D. J. Hinde, M. Dasgupta, J. R. Leigh, J. C. Mein, C. R. Morton, J. O. Newton et al., Conclusive evidence for the influence of nuclear orientation on quasifission, Phys. Rev. C 53 (Mar, 1996) $1290-1300$.

[15] K. Hammerton, Z. Kohley, D. J. Hinde, M. Dasgupta, A. Wakhle, E. Williams et al., Reduced quasifission competition in fusion reactions forming neutron-rich heavy elements, Phys. Rev. C 91 (Apr, 2015) 041602.

[16] C. J. Lin, R. du Rietz, D. J. Hinde, M. Dasgupta, R. G. Thomas, M. L. Brown et al., Systematic behavior of mass distributions in ${ }^{48}$ ti-induced fission at near-barrier energies, Phys. Rev. C 85 (Jan, 2012) 014611.

[17] Yu. Ts. Oganessian, V. K. Utyonkov, Y. V. Lobanov, F. S. Abdullin, A. N. Polyakov, R. N. Sagaidak et al., Synthesis of the isotopes of elements 118 and 116 in the ${ }^{249} \mathrm{cf}$ and ${ }^{245} \mathrm{~cm}+{ }^{48}$ ca fusion reactions, Phys. Rev. C 74 (2006) 044602.

[18] E. V. Prokhorova, A. A. Bogachev, M. G. Itkis, I. M. Itkis, G. Knyazheva, N. A. Kondratiev et al., The fusion-fission and quasi-fission processes in the reaction $48 \mathrm{ca}+208 \mathrm{pb}$ at energies near the coulomb barrier, Nucl. Phys. A 802 (2008) 45 - 66.

[19] D. J. Hinde and M. Dasgupta, A new framework to investigate the systematics of fusion probabilities in heavy element formation: Application to th isotopes, Phys. Lett. B 622 (2005) 23 - 28. 